\title{
A ENFERMAGEM NO CUIDADO DA CRIANÇA AUTISTA NO AMBIENTE ESCOLAR
}

\section{Bruna Sabrina de Almeida Sousa}

Enfermeira. Especialização em Saúde Pública e Docência do Ensino Superior. Faculdade Evangélica do Meio Norte (FAEME). Teresina, Piauí, Brasil.

\section{Camila Aparecida Pinheiro Landim Almeida}

Enfermeira. Doutora em Ciências pela Escola de Enfermagem de Ribeirão Preto da Universidade de São Paulo (EERP/USP). Ribeirão Preto. Docente Titular do Programa de Mestrado Profissional em Saúde da Família do Centro Universitário UNINOVAFAPI. Teresina Piauí, Brasil.

\section{Herica Emilia Félix de Carvalho}

Enfermeira. Mestranda do Programa de Pós-Graduação em Enfermagem. Universidade Federal do Piauí. Teresina, Piauí, Brasil.

Enfermeira. Mestranda do Programa de Pós-Graduação em Enfermagem. Universidade Federal do Piauí. Teresina, Piauí, Brasil.

\section{Jardel Nascimento da Cruz}

Discente do $8^{\circ}$ período do Curso de Graduação em Bacharelado em Enfermagem. Centro Universitário UNINOVAFAPI. Teresina, Piauí, Brasil.

\section{Lorrainie de Almeida Gonçalves}

RESUMO: Este estudo objetivou descrever uma reflexão acadêmica acerca da enfermagem no cuidado da criança autista no ambiente escolar. Estudo descritivo, do tipo relato de experiência, baseado em uma vivência de estágio acadêmico, de agosto a dezembro de 2016. Os resultados foram apresentados de forma descritiva em duas categorias temáticas: o primeiro contato e o acompanhamento com a criança autista; e dificuldades encontradas no cuidado prestado à criança autista em ambiente escolar. A experiência vivenciada superou as expectativas pré-estabelecidas para um estágio, tendo em vista que os maiores benefícios desta experiência foram a oportunidade gratificante de zelar diariamente pela saúde biopsicossocial de uma criança autista, o desenvolvimento de um valoroso elo de empatia com a criança, além da relação de troca de conhecimentos e relações interpessoais com os demais alunos e profissionais da unidade escolar.

PALAVRAS-CHAVE: Cuidados de enfermagem; Criança; Transtorno autístico; Educação infantil.

\section{NURSING IN AUTISTIC CHILDREN'S CARE WITHIN THE SCHOOL ENVIRONMENT}

\begin{abstract}
Current descriptive analysis reports on nursing in autistic children's care within the school environment. The report was based on the experience of a student between August and December 2016. Results were presented in two thematic categories: first contact and the following-up of an autistic child; difficulties in care giving to autistic children within the school environment. Experience went beyond the pre-established expectations for a traineeship. In fact, the greatest asset of the experience was the opportunity to daily care giving for the bio-psycho-social health of the autistic child, the development of a bond with the child, exchange of information and interpersonal relationships with the other students and professionals of the school.
\end{abstract}

KEY WORDS: Nursing care; Children; Autistic disorder; Children's education.

\section{INTRODUÇÃO}

O autismo é classificado como um transtorno global do deAutor correspondente

Camila Aparecida Pinheiro Landim Almeida

E-mail: camila@uninovafapi.edu.br senvolvimento, caracterizando-se pelo desenvolvimento atípico da interação social, e da presença de um repertório restrito, com atrasos significativos na comunicação evidenciados pela linguagem expressiva limitada, dificuldades com reciprocidade social e movimentos estereo- 
tipados e repetitivos; com resistência a mudanças, incapacidade de desenvolver um contato olho a olho antes dos cinco anos de idade, e respostas anormais ao estímulo visual e auditivo. ${ }^{1-4}$

Trata-se de disfunção psiconeurológica no desenvolvimento, no qual os déficits podem ser manifestados de forma leve, moderada e grave, e de acordo com alguns estudos, com incidência maior em crianças do sexo masculino do que feminino, com proporção de quatro indivíduos do sexo masculino para cada um do sexo feminino; quando ocorre em meninas, geralmente, os sinais e sintomas apresentam-se com um grau de severidade mais acentuado. ${ }^{2,5,6}$

Com base no último relatório dos Centros para Controle e Prevenção de Doenças, um em cada 68 nascimentos foi uma criança autista nos Estados Unidos em 2014. ${ }^{7}$ Até o ano 2020, os distúrbios neuropsiquiátricos na infância aumentarão em mais de 50\% em nível internacional para se tornar uma das cinco causas mais comuns de morbidade e deficiência entre as crianças. ${ }^{8}$ De acordo com a Associação Americana Psiquiátrica, em relação aos indivíduos diagnosticados com Transtorno do Espectro Autista (TEA), são caracterizados três níveis com base no suporte necessário: nível 1, exigindo suporte; nível 2, que requer apoio substancial; e nível 3, exigindo suporte extremo em todos os momentos. ${ }^{1}$

A Educação Especial surgiu no final do século XVIII e início do XIX nos países escandinavos e América do Norte. No Brasil, desde o Império existiram instituições com educação caracterizada pela segregação. Somente a partir de 1960, começou a ser questionada essa maneira de educar, havendo uma luta para que as crianças pudessem aprender junto às escolas regulares, de maneira a poder integrá-las. ${ }^{9}$

Então, no Brasil, promulgou-se, em 2008, a Política Nacional de Educação Especial na perspectiva da educação inclusiva, reafirmando o direito de todos os alunos frequentarem o sistema regular de ensino. Essa política nacional orienta os sistemas de ensino quanto à inclusão de alunos com deficiência, transtornos globais do desenvolvimento e altas habilidades/superdotação. ${ }^{10}$

A criança portadora do autismo, com base na educação inclusiva, necessita de auxílio e estímulo contínuo, principalmente no ambiente escolar, que durante a fase estudantil, é onde as crianças passam boa parte do tempo. ${ }^{3}$ De acordo com a lei Berenice Piana de $n^{0} 12.764$, de 27 de dezembro de2012, no artigo $3^{\circ}$ no inciso IV são direitos da pessoa com transtorno do espectro autista ter acesso à educação e ensino profissionalizante. O parágrafo único ainda ressalta que em casos de comprovada necessidade, a pessoa com autismo incluída nas classes comuns de ensino regular, tem direito a acompanhante especializado., ${ }^{2,6,11}$

Perante à necessidade de acompanhamento e cuidado à criança autista, a enfermagem dispõe de todo conhecimento prático e científico para auxiliar as crianças com autismo a tornarem-se indivíduos ativos na construção de sua vida e de sua independência. A enfermagem, no contexto de educar, pode atuar na educação especial promovendo o desenvolvimento das potencialidades das pessoas com deficiência em todo o aspecto biopsicossocial. $^{4}$

Frente à contextualização científica apresentada, o presente estudo motivou-se pela expectativa em provocar discussões construtivas sobre o cuidado de enfermagem às crianças autistas, em meio ao ambiente escolar. Para a prática profissional, o estudo torna-se extremamente relevante e poderá ser utilizado como subsídios para compreender a importância do cuidado de enfermagem no TEA.

Para este estudo, selecionou-se como método o estudo descritivo, do tipo relato de experiência. Os relatos de experiência são considerados metodologias de observação sistemática da realidade, sem a intenção de testar hipóteses, mas estabelecendo relações entre os achados da realidade com bases teóricas pertinentes. ${ }^{12}$

A obtenção do material empírico ocorreu por meio da interpretação e descrição da experiência vivenciada no período de agosto a dezembro de 2016, em uma unidade escolar da prefeitura municipal de Teresina, capital do Piauí, por meio da vivência de um estágio profissional designado para a função do acompanhamento em saúde de uma criança portadora do Transtorno do Espectro Autista, cursando o primeiro ano do ensino fundamental. Este estágio profissional foi oportunizado pela prefeitura municipal de Teresina e ocorreu nos turnos das tardes, das $12 \mathrm{~h} 00 \mathrm{~min}$ às $18 \mathrm{~h} 00 \mathrm{~min}$, sendo 30 horas semanais e perfazendo um total de 600 horas. 
Em conformidade com os preceitos da resolução $\mathrm{n}^{0}$ 510, de 07 de abril de 2016, do Conselho Nacional de Saúde, que trata de especificidades éticas de pesquisas nas ciências humanas e sociais, o presente estudo possibilita o aprofundamento teórico de situações que emergiram espontaneamente e contingencialmente na prática profissional. Portanto, não há exposição e publicação de dados que possam identificar o participante envolvido; sendo dispensada a apreciação por um Comitê de Ética em Pesquisa. ${ }^{13}$

Dessa forma, este estudo objetivou descrever uma reflexão acadêmica acerca da enfermagem no cuidado da criança autista no ambiente escolar. Os resultados desta reflexão acadêmica foram apresentados de forma descritiva por meio do desenvolvimento de duas categorias temáticas: o primeiro contato e o acompanhamento com a criança autista; e, dificuldades encontradas no cuidado prestado à criança autista em ambiente escolar.

\section{DESENVOLVIMENTO}

\section{O PRIMEIRO CONTATO E O ACOMPANHAMENTO COM} A CRIANÇA AUTISTA

A experiência a ser descrita em relação ao acompanhamento em saúde, iniciou-se no mês de agosto de 2016, por meio da classificação para um estágio profissional voltado ao auxílio pedagógico em saúde para uma criança matriculada no primeiro ano do ensino fundamental do ensino regular de uma unidade escolar da prefeitura municipal de Teresina, capital do Estado do Piauí, Brasil.

A criança a qual foi encaminhado o acompanhamento, foi do sexo masculino, com seis anos de idade. Tais informações corroboram com estudos que afirmam que há maior incidência de TEA em crianças do sexo masculino, e que os sintomas iniciam-se ainda na infância. $^{2,5,6,14-16}$

A referida criança havia sido diagnosticada com o Transtorno do Espectro Autista há apenas oito meses. Segundo informações da professora responsável pelo Atendimento Educacional Especializado (AEE) e familiares, a criança apresentava TEA grau leve (nível 1). O diag- nóstico, de acordo com Associação Americana Psiquiátrica, e o Manual Diagnóstico e Estatístico de Transtornos Mentais (DSM-V), foi classificado como nível 1, pelo grau de necessidade de suporte e características dos déficits e padrões comportamentais. ${ }^{1,9}$

Seguindo os critérios diagnósticos, o laudo médico é baseado em observações comportamentais e em informações relatadas pelos pais. A partir da observação das características do comportamento, é possível classificar a gravidade, mensurar progressos ou retrocessos e programar intervenções. ${ }^{17} \mathrm{Com}$ isso, foi solicitado à criança, por meio da secretaria municipal de educação, uma auxiliar de apoio e inclusão para o acompanhamento em saúde.

Durante os primeiros contatos no ambiente escolar, o foco inicial era conhecer a rotina da criança, sua personalidade e dificuldades, a fim de reconhecer suas verdadeiras necessidades, e possibilitar a aproximação com cautela e respeito ao espaço da criança, pois, até então, a mesma não teria ainda compreendido a ideia de que teria um acompanhamento e como ocorreria este acompanhamento dia após dia. O reconhecimento das necessidades da criança ocorreu por meio da identificação de dificuldades percebidas no cotidiano. Observou-se a linguagem oral e corporal, a comunicação e socialização com os demais colegas, o humor, o comportamento e o nível de atenção, a fim de se conhecer as especificidades da criança.

A princípio, nos primeiros dias de estágio na unidade escolar, a proposta foi conhecer o ambiente escolar e não só a criança a qual a pesquisadora foi designada para os cuidados, mas também conhecer as demais crianças e adolescentes que foram diagnosticadas com deficiências intelectuais, assim como as que estavam aguardando o diagnóstico. Dessa forma, durante os primeiros dias, teve-se a oportunidade de conhecer como funciona o acompanhamento pedagógico, através do apoio e auxílio da professora de AEE.

$\mathrm{O}$ atendimento educacional especializado - AEE tem como função identificar, elaborar e organizar recursos pedagógicos e de acessibilidade que eliminem as barreiras para a plena participação dos alunos, considerando suas necessidades específicas; é realizado, prioritariamente, na sala de recursos multifuncionais da própria escola 
ou em outra escola de ensino regular, no turno inverso da escolarização, podendo ser realizado, também, em centro de atendimento educacional especializado. ${ }^{18}$

A unidade escolar onde ocorreu a experiência era composta por professores, coordenadores, diretores, motoristas, monitores, porteiros, auxiliares de serviços gerais e cozinheiras, assim como a função a qual ocupou a pesquisadora, como auxiliar de apoio e inclusão para o acompanhamento em saúde. No turno da manhã, havia mais crianças diagnosticadas com deficiências intelectuais, sendo que no turno da tarde, apenas a criança a qual a pesquisadora foi designada, possuía acompanhante.

A lei $n^{\circ} 12.764$ ressalta o acesso a ações e serviços de saúde, com vistas à atenção integral às necessidades de saúde da pessoa com TEA, e garante ao aluno com autismo o direito à matrícula no ensino regular, o atendimento educacional especializado realizado no contraturno, e um profissional de apoio, disponibilizado pelo sistema de ensino, sempre que identificada a necessidade de acompanhamento. A função de acompanhante baseia-se no cuidado holístico, com assistência voltada à autonomia e progresso dos aspectos biopsicossociais da pessoa com TEA. ${ }^{10,11}$

O acompanhamento descrito neste relato ocorreu de segunda-feira à sexta-feira, nos turnos das tardes, em todos os horários de aulas. Durante o horário do recreio, a supervisão era a distância, a fim de oferecer maior liberdade à criança. No momento da aula, a criança sentava ao meu lado, com meus cuidados destinados a ajudá-la em suas atividades, desenvolvimento de expressão, linguagem, leituras e escrita, participações durante a aula, auxílio em saúde, quando necessários, e esforços para minimizar suas dificuldades e limitações.

Como um profissional inserido na equipe de cuidado à saúde de uma criança com TEA, o enfermeiro deve ter preparação para intervir junto à criança e sua família, adotar uma abordagem teórica de enfermagem que possibilite à criança com autismo o autocuidar-se de acordo com seu potencial e limitação, para que possa então ter autonomia em sua vida diária. ${ }^{1,20}$

Dessa forma, torna-se essencial que o enfermeiro esteja capacitado para prestar o cuidado lúdico e, ao mesmo tempo, seguro. A formação humanística do en- fermeiro é condição primordial para o acompanhamento dessas crianças. ${ }^{21,22}$

Sempre estar atento aos sinais e sintomas do autismo e sabendo diferenciar as demais síndromes, proporcionando boa assistência de enfermagem à criança e a seus pais, encorajando, transmitindo segurança e tranquilidade a todos. Incentivar os pais no tratamento de seus filhos e orientá-los a se unirem a grupos de pais que estão passando por situações parecidas, para juntos compartilharem experiências vivenciadas.

A criação de vínculo entre o profissional, a criança e as famílias é fundamental. Com isso, tanto o paciente quanto os pais se sentirão seguros e poderão ajudar no tratamento. Criar atividades de interação entre toda a família, como brincadeiras e danças ajudará na interação social. Para isso, há grande necessidade de novos estudos e investigações para que contribuam no desenvolvimento e ampliação de um olhar clínico da assistência de enfermagem e dos demais profissionais.

\section{DIFICULDADES ENCONTRADAS NO CUIDADO PRESTA- DO À CRIANÇA AUTISTA EM AMBIENTE ESCOLAR}

Inicialmente, uma das dificuldades encontradas, em relação ao acompanhamento à criança autista, referiu-se à aproximação, pois, o universo infantil possui suas particularidades, em que a entrada de um adulto desconhecido para o convívio diário pode representar um desafio para a realização efetiva do acompanhamento em saúde.

Foram encontradas barreiras para a aproximação com a criança, pela sua própria personalidade característica, que apresentava dificuldades na interação social, relacionadas às manifestações de comportamento estereotipado e impulsivos, a recusa em manter contato visual, recusa em falar, apresentando comportamentos como a agressividade, o alheamento e hiperatividade. ${ }^{3,15}$

A principal estratégia para romper tal barreira de aproximação foi manter o contato de forma gradual, respeitando o tempo de adaptação da criança e sua forma de reagir a um primeiro contato. A fim de estabelecer uma afinidade, foi exercida uma postura acolhedora baseada na subjetividade, evidenciando o anseio de manter 
um vínculo benéfico para a criança, apoiando-a em suas necessidades, respeitando seu espaço e auxiliando nas dificuldades com compreensão, descrição e respeito.

Por vezes, a criança expressava dificuldades para manter a atenção, hiperatividade, impulsividade e agressividade física e/ou verbal com professores e alunos da escola. Em alguns momentos esquivava-se de manter contato com outras pessoas, demonstrando insatisfação com o meio; o que vai de encontro com estudos que evidenciam as características impulsivas, estereotipadas, hiperativas e os déficits sociais de crianças com TEA.3,10,14,15

Durante a aula, tais características exacerbavam-se quando ele não conseguia resolver suas atividades, conversar com crianças da sala, ou mesmo quando sentia vontade de ficar recluso no ambiente. Para tanto, foram utilizadas as estratégias de desenhos como meio para a socialização da criança, e ela poderia compartilhar seus desenhos com outras crianças da sala e poderia se expressar em forma de desenho suas vontades e intenções.

O momento da leitura também foi de suma importância, pois neste momento, o foco foi a interação do pesquisador, por meio do exercício de suas capacidades intelectuais, como a imaginação, o raciocínio, a leitura e a memória. Foi um momento de descontração, no qual se perceberam resultados extremamente benéficos para a alfabetização da criança, desenvolvimento cognitivo, intelectual e sociabilidade. A estimulação das capacidades sociais da criança por meio de materiais lúdicos é um fator positivo para o aprimoramento de habilidades e desenvolvimento cognitivo.

Rocha et al.23 destacam a importância de realizar o acompanhamento da maturidade cognitiva em pré-escolares, tendo em vista a possibilidade de interferir na capacidade de executar atividades físicas e motoras. Dessa forma, compreende-se que o acompanhamento da maturidade cognitiva pode ser um fator facilitador no acompanhamento de crianças com TEA no ambiente escolar.

Além disso, crianças dentro do espectro autista geralmente precisam de apoios visuais e o sistema de comunicação por troca de figuras (PECS - Picture Exchange Comunication System), que trabalham justamente o aprendizado da comunicação.9

Apesar da existência de estratégias que estimu- lem as crianças com TEA, existem ainda entraves que dificultam a permanência ativa dessas crianças no ensino regular como, por exemplo, a falta de conhecimento de professores em como efetivar o ensino e a acessibilidade, com materiais didáticos e objetos adaptados. O professor não se sente responsável e capacitado pela adequação dos conteúdos para o aluno com autismo, delegando essa atividade às acompanhantes e/ou estagiárias.6

Tal situação ocorreu continuadamente nesta experiência, pois a professora responsável pela turma não realizava adequações de material para a criança com autismo, deixando tal função apenas para a professora de AEE, sobrecarregando-a, corroborando com estudos que mostram como as adequações curriculares, ainda não fazem parte da realidade cotidiana das salas de aula.6 As dificuldades na escola e a escassez de profissionais especializados em oferecer uma educação direcionada estão realmente comprometendo o aprendizado. 2

A realidade do ambiente escolar deve ser adequada ao favorecimento do desenvolvimento e aprendizado da criança, pois o contexto escolar propicia contatos sociais, o que favorece o desenvolvimento da criança autista, na medida em que convivem e aprendem com as diferenças.3 No entanto, a unidade escolar em questão, não possuía infraestrutura favorável para o bem-estar dos alunos, sendo uma escola com estrutura deficitária e baixa segurança.

O escasso recurso humano, de material permanente e financeiro, limitações e fragilidades percebidas e vivenciadas no cotidiano da escola, infraestrutura física deteriorada, dificultam a ambiência dos alunos, principalmente os alunos com deficiências. ${ }^{3}$

Além disso, pôde-se visualizar a ocorrência de quedas e lesões nas crianças menores, inclusive nas crianças com deficiência intelectual, pela infraestrutura de risco do colégio e a junção de adolescentes e crianças menores, no mesmo intervalo recreativo. As deficiências estão relacionadas não somente com a condição individual e intrínseca da pessoa, mas também à sua relação com o ambiente social (ROSSO; LOSSO, 2016). ${ }^{4}$

A família representa outro fator entendido como dificuldade, pois a falta de comparecimento da família no ambiente escolar representa um problema para o acompanhamento em saúde. Por motivos de trabalho 
e falta de tempo, os pais da criança acompanhada referiram não poder ir à escola constantemente, mesmo sendo preocupados com as necessidades, progressos e cotidiano da criança.

A literatura revelou que os pais de crianças com TEA experimentam níveis elevados de estresse, depressão e ansiedade. A família se sente incapaz diante do desafio de ajustar seu tempo, planos e expectativas quanto ao futuro e às necessidades da criança, além da necessidade de adaptar-se à intensa dedicação e prestação de cuidados com o filho. As mães costumam experimentar mais estresse do que os pais, por serem as principais cuidadoras das crianças com TEA., 2,7,24

Em suma, construir uma escola que tenha por base não a homogeneidade, mas a diversidade, é o grande desafio que se precisa enfrentar na atualidade, pois a educação inclusiva não se fará se não forem introduzidos na sala de aula instrumentos diferentes e adaptados; a luta da população com autismo não é mais pelo ingresso nas escolas da rede regular de ensino, pois por lei ele já está garantido. A luta a ser travada é quanto à garantia da educação de qualidade. ${ }^{6}$

\section{CONSIDERAÇÕES FINAIS}

A experiência vivenciada no cuidado de enfermagem à criança autista no ambiente escolar superou as expectativas estabelecidas para o estágio profissional em questão, tendo em vista que o maior benefício desta experiência foi a oportunidade gratificante de zelar diariamente pela saúde biopsicossocial de uma criança autista. Além disso, a partir do convívio diário, foi construído um importante elo de empatia e sentimento pessoal com a criança.

Dentre os benefícios obtidos a partir do acompanhamento em saúde relatado neste estudo, destacaram-se: a melhoria do desenvolvimento social da criança; aprimoramento da leitura e escrita, bem como participação durante a aula; melhora da linguagem e expressão, e diminuição da irritabilidade. Cabe ressaltar sobre a necessidade de treinamento e capacitação de professores, educadores e profissionais de saúde, inclusive os enfermeiros, a fim de estabelecerem estratégias adapta- tivas para o desenvolvimento de crianças com TEA, vislumbrando a conquista da autonomia para a inserção no ensino regular no meio social.

\section{REFERÊNCIAS}

1. Dillon SR, Adams D, Goudy L, Bittner M, Mcnamara S. Evaluating Exercise as Evidence-Based Practice for Individuals with Autism Spectrum Disorder. Front Public Health [internet] 2016 Feb [cited 2017 jun 12];4(290)1-18. Available from: https://www.ncbi. nlm.nih.gov/pmc/articles/PMC5293813/ doi: 10.3389/ fpubh.2016.00290

2. Maia filho ALM, Nogueira LANM, Nogueira AL, Silva KCO, Santiago RF. A importância da família no cuidado da criança autista. Rev. Saúde em Foco [internet] 2016 jan-jun [acesso em 2017 jun 11];3(1):66-83. Disponível em: http://www4.fsanet.com.br/revista/index. $\mathrm{php} / \mathrm{saudeemfoco/article/view/719}$

3. Mesquita LP, Pena JLC. Crenças do professor sobre autismo e inclusão na educação infantil. Extensão em Ação [internet] 2016 jul-out [acesso em 2017 jun 11];2(11): 25-38. Disponível em: http://www.revistaprex.ufc.br/index.php/EXTA/article/view/324/168

4. Rosso LE, Losso ARS. Cuidado de enfermagem na APAE: necessidades da equipe multiprofissional. Inova Saúde [internet] $2016 \mathrm{dez}$ [acesso em 2017 jun 12];5(2): 45-62. Disponível em: http://periodicos. unesc.net/Inovasaude/article/view/3020/2790 doi: http://dx.doi.org/10.18616/is.v5i2.3020

5. Bortone ART, Wingester ELC. Identificação do espectro do transtorno autista durante o Crescimento e o desenvolvimento infantil: o papel do profissional de enfermagem. SynThesis FAPAM [internet] $2016 \mathrm{dez}$ [acesso em 2017 jun 10];7(7): 131-48. Disponível em: http://periodicos.fapam.edu.br/index.php/synthesis/ article/viewFile/133/130.

6. Sanches IR, Siqueira LPC. A inclusão escolar e o transtorno do espectro do autismo. Comunicações [internet] 2016 [acesso em 2017 jun 11];23(3): 
167-83. Disponível em: https://www.metodista.br/ revistas/revistas-unimep/index.php/comunicacoes/article/view/2926/1861 doi: http://dx.doi.org/10.15600/ 2238-121X/comunicacoes.v23nespp167-183

7. Hajiabolhasani-Nargani Z, Najafi M, Mehrabi T. Effect of mobile parenting skills education on anxiety of the mothers with autistic children. Iran J Nurs Midwifery Res. [internet] 2016 Nov-Dez [cited 2017 Jun 18] ;21(6): 572-6. Available from: https://www. ncbi.nlm.nih.gov/pmc/articles/PMC5301062/ doi: 10.4103/1735-9066.197668.

8. Daniel LT, Gupta S, Sagar R. Effect of Educational Module on Knowledge of Primary School Teachers Regarding Early Symptoms of Childhood Psychiatric Disorders. Indian J Psychol Med. [internet] 2013 Out-Dez [cited 2017 Jun 20];35(4): 368-72. Available from: https://www.ncbi.nlm.nih.gov/pmc/articles/ PMC3868088/\#!po $=4.54545$ doi: $10.4103 / 0253$ 7176.122228 .

9. Silva MM. Educação especial na perspectiva inclusiva: crianças com transtorno do espectro do autismo. Id on Line Rev Psic. [internet] $2017 \mathrm{fev}$ [acesso em 2017 jul 15];11(35): 56-66. Disponível em: https://idonline. emnuvens.com.br/id/article/view/717/1049

10. Guareschi T, Alves MD, Naujorks MI. Autismo e políticas públicas de inclusão no Brasil. J Res Spec Educ Needs [internet] 2016 ago [acesso em 2017 jun 12];16(1): 246-50. Disponível em: http://onlinelibrary.wiley.com/doi/10.1111/1471-3802.12286/full doi: 10.1111/1471-3802.12286.

11. Brasil. Presidência da República. Congresso Nacional. Lei ${ }^{0}$ 12.764, de 27 de dezembro de 2012. Política Nacional de Proteção dos Direitos da Pessoa com Transtorno do Espectro Autista. [internet] 2012 [acesso em 2017 jun 11]. Disponível em: http://www.planalto. gov.br/ccivil_03/_ato2011-2014/2012/lei/112764.htm.

12. Lima GK, Dias ICX, Araújo FM, Souza SB, Sales DS, Ferreira AGN. Educação em saúde sobre sífilis com um grupo de gestantes: um relato de experiência de acadêmicas de enfermagem. Sanare [internet] 2013 jun-dez [acesso em 2017 jun 18];12(2): 59-62. Dis- ponível em: https://sanare.emnuvens.com.br/sanare/ article/view/384/276

13. Brasil. Conselho Nacional de Saúde. Resolução $n^{0}$ 510, de 07 de abril de 2016. Pesquisa na área de Ciências Humanas e Sociais. [internet] 2016 [acesso em 2017 jun 20]. Disponível em: http://conselho.saude. gov.br/resolucoes/2016/Res0510.pdf.

14. Ebertb M, Lorenzinic E, Silva EF. Mothers of children with autistic disorder: perceptions and trajectories. Rev Gaúcha Enferm. [internet] 2015 Mar [cited 2017 Jun 12];36(1): 49-55. Available from: http:// www.scielo.br/scielo.php?script =sci_arttext\&pi$\mathrm{d}=$ S1983-14472015000100049\&lng=en doi: http:// dx.doi.org/10.1590/1983-1447.2015.01.43623.

15.Guareschi T, Alves MD, Naujorks MI. Políticas públicas e educação especial: uma análise sobre o autismo. Atos de Pesquisa em Educação [internet] 2016 ago-nov [acesso em 2017 jun 11];11(2): 374-95. Disponível em: http://proxy.furb.br/ojs/index.php/atosdepesquisa/article/view/4903/3329 doi: http://dx.doi. org/10.7867/1809-0354.2016v11n2p374-395.

16. Nogueira MAA, Rio SCMM. A família com criança autista: apoio de enfermagem. Rev. port. enferm. saúde mental [internet] 2011 jun [acesso em 2017 jun 11] ; (5):16-21. Disponível em: http://www. scielo.mec.pt/scielo.php?script $=$ sci_arttext\&pi$\mathrm{d}=$ S1647-21602011000100003\&lng $=\mathrm{pt}$

17. Campos LK, Fernandes FDM. Perfil escolar e as habilidades cognitivas e de linguagem de crianças e adolescentes do espectro do autismo. CoDAS [internet] 2016 jun [acesso em 2017 jun 12];28(3): 234-43. Disponível em: http://www.scielo.br/scielo.php?script $=$ sci_arttext\&pid $=$ S2317-17822016000300234\&lng $=$ en doi: http://dx.doi.org/10.1590/2317-1782/20162015023.

18. Ministério da Educação (BR). Conselho Nacional de Educação, Câmara de Educação Básica. Diretrizes operacionais da educação especial para o atendimento educacional especializado na educação básica. [internet] 2009 [acesso em 2017 jun 11]. Disponível em: http://portal.mec.gov.br/dmdocuments/rceb004_09. 
pdf.

Recebido em: 26/07/2017

19. Carniel EL, Saldanha LB, Fensterseifer LM. A atuação Aceito em: 22/02/2018 do enfermeiro frente à criança autista. Pediatria (São Paulo) [internet] 2010 out-dez [acesso em 2018 jan 26];32(4): 255-60. Disponível em: http://www.pediatriasaopaulo.usp.br/upload/pdf/1361.pdf

20. Abreu e Andrade A, Teodoro MLM. Família e Autismo: Uma Revisão da Literatura. Contextos Clínic. [internet] $2012 \mathrm{dez}$ [acesso em 2018 jan 26];5(2): 133-42. Disponível em: http://pepsic.bvsalud.org/scielo.php?script $=$ sci_arttext\&pid $=$ S1983-34822012000200008 doi: $10.4013 /$ ctc.2012.52.07

21. Franzoi MAH, Santos JLG, Backes VMS, Ramos FRS. Intervenção musical como estratégia de cuidado de enfermagem a crianças com Transtorno do Espectro do Autismo em um centro de atenção psicossocial. Texto Contexto Enferm. [internet] 2016 [acesso em 2018 jan 26];25(1):1-8 Disponível em: http://www.scielo. br/pdf/tce/v25n1/0104-0707-tce-25-01-1020015.pdf

22. Pereira DVM, Durães DTS, Caiado FL, Sales JWB, Figueiredo MFL, Barbosa MR. Inclusão de crianças autistas no ambiente escolar: uma revisão de literatura. Renome [internet] 2015 [acesso em 2018 jan 26];4(spe): 15-6. Disponível em: http://www.renome. unimontes.br/index.php/renome/article/view/31/31

23. Rocha FF, Santos VAP, Contreira AR, Pizzo GC, Silva PN, Romero PVS, et al. Análise do desempenho motor e maturidade cognitiva de pré-escolares de Maringá (PR). Revista Saúde e Pesquisa [internet] 2016 setdez [acesso em 2017 jun 12];9(3): 507-15. Disponível em: http://periodicos.unicesumar.edu.br/index.php/ saudpesq/article/view/5448/2918 doi: http://dx.doi. org/10.177651/1983-1870.2016v9n3p507-515

24. Rayan A, Ahmad M. Psychological Distress in Jordanian Parents of Children with Autism Spectrum Disorder: The Role of Positive Reappraisal Coping. Arch Psychiatr Nurs. [internet] $2017 \mathrm{Feb}$ [cited 2017 Jun 20];31(1): 38-42. Available from: http://www.psychiatricnursing. org/article/S0883-9417(16)30147-9/fulltext doi: http:// dx.doi.org/10.1016/j.apnu.2016.07.017 